\title{
The use of perspective as a depth cue with a 2D display in 4 and 5-month-old infants
}

\author{
Myriam Frichtel, Roger Lécuyer* \\ Laboratoire Cognition et Comportement, Université René Descartes/CNRS, 71 Avenue Edouard Vaillant, \\ 92774 Boulogne-Billancourt, Cedex, France
}

Received 23 July 2005; received in revised form 19 June 2006; accepted 17 November 2006

\begin{abstract}
Four experiments were conducted to study the use of perspective as a depth cue in infants, using an eye-tracking-system. In the first experiment, no significant difference was observed between the looks for the "normal" and the "strange" events on the complete display and at the target in 4-month-olds. In the second experiment, the results of 5-month-olds were similar to those obtained by 4-month-olds but they looked more at the test events when the "strange" event was presented first. In the third experiment, 5 month-olds were shown a repeated presentation adapted from the "Partial-Lag" design. Infants' exploration of the target indicated that they looked more at the "strange" event than at the "normal" event. In the fourth experiment, the same design was used with 4-month-olds but no difference between conditions was observed. Five-month-olds seem to be able to use the perspective cues alone. These different data are discussed.
\end{abstract}

(C) 2007 Elsevier Inc. All rights reserved.

Keywords: Infant; Representation; Visual; Depth; Perspective; 2D/3D

\section{Introduction}

Several experiments have been carried out about the perception of depth. Gibson and Walk (1960) are the first authors to show that 9-month-old infants perceive the depth on a visual cliff paradigm. Campos, Langer, and Krowitz (1970) evidenced this capacity in 2-month-olds. Fantz (1961), replicated by Fantz and Nevis (1967), using another paradigm, demonstrated that young infants discriminate 2D and 3D stimuli with a preference for 3D ones.

Yonas has studied the emergence of sensitivity and responsiveness to different depth cues of the third dimension with kinetic, binocular and pictorial information (see, Yonas \& Owsley, 1987, for a review). According to Yonas and Granrud (1985), the mechanisms that detect kinetic, binocular and pictorial information function are three distinct systems. Their research demonstrates a development of the capacity to implement these different cues, from birth to the second half of the first year. They suggest that sensitivity to kinetic, binocular and pictorial information develops in a fixed sequence. Some sensitivity to kinetic information may be present at birth or soon thereafter as at one month (Nanez \& Yonas, 1994); sensitivity to binocular depth information would appear during the fourth month between 3 and 5 months of age, (see, Yonas, Arterberry, \& Granrud, 1987); and sensitivity to static monocular depth cues (pictorial

\footnotetext{
* Corresponding author. Tel.: +33155205990.

E-mail addresses: myfricht@yahoo.fr (M. Frichtel), roger.lecuyer@ psycho.univ-paris5.fr (R. Lécuyer).
} 
information) would appear between five and seven months of age (Granrud, Yonas, \& Pettersen, 1984; Maya, Yonas, \& Knill, 2001; Yonas \& Arterberry, 1994; Yonas, Cleaves, \& Pettersen, 1978). Nevertheless, the infants' responsiveness to static pictorial information is proved in 7-month-olds and in 6-month-olds in these experiments but this capacity is not evidenced in 5-month-olds except in a longitudinal research where the variability was shown at the age when infants start using linear perspective and texture gradient for perceiving depth (22-28 weeks of age), and infant's sensitivity emerged across 2-8 weeks (Yonas, Elieff, \& Arterberry, 2002).

These results were found in using a manual reaching gesture but this use of a motor response to evaluate infant cognition may be the source of an important bias (Mandler, 1998). The use of a manual motor response in most of these experiments may have led to an underestimation of infants' capacity to understand depth cues as observed in the relations between motor responses and other aspects of infant cognition (Baillargeon, Spelke, \& Wasserman, 1985; Lécuyer, Pêcheux, \& Streri, 1994). The sensitivity to pictorial information could be detected early with a visual paradigm. Using a purely visual situation with moving stimuli and a fixation duration index, Lécuyer and Durand (1998) tested 4-month-old infants' capacity to understand interposition, replicating Baillargeon and Graber (1987) and Baillargeon and DeVos's (1991) experiments in a 2D display involving a television screen. The permanence of object was found in infants aged 5.5 and 3.5 months with the 3D situation but this result was not observed in 4-month-olds with the $2 \mathrm{D}$ representation, suggesting that in a complex situation more perceptual indices must be given to infants. As this situation was cognitively complex, a simpler interposition situation was displayed to 3-month-olds in 2D representation and the results showed that infants are sensitive to dynamic interposition.

In another research, Durand and Lécuyer (2002), showed in a 2D replication of the experiment by Baillargeon et al. (1985) that 4-month-olds understand the object permanence and dynamic interposition when perspective cues are present.

The question is to know if infants are able to perceive depth in a 2D display when perspective is the only available cue. In order to answer this question, three experiments were carried out by Durand, Lécuyer, and Frichtel (2003), in using a habituation/violation of expectation paradigm to test if infants understand the perspective rule in which the object's size varies as a function of its apparent distance. These experiments refer to the results of Slater, Mattock, \& Brown (1990) who demonstrated size constancy in newborns in a 3D situation and we wanted to determine if infants can take into account the same relation in a $2 \mathrm{D}$ display.

In the first experiment, 3-month-olds were asked to extrapolate the rule of the perspective "apparently further away then smaller" with a sequential presentation of this rule in the habituation phase with a 2D display involving a television screen. Infants were habituated to a cube displayed at different apparent distances with different sizes related to these apparent distances in a background with perspective cues. After habituation, they were confronted with an extrapolation of this rule in a "normal situation" (the cube was apparently further away and smaller) alternated with a violation of the rule in a "strange situation" (the cube was apparently away but its size was the mean size of the habituation stimuli). No difference appeared in looking durations for the normal and the strange event and none of the results obtained indicated such an extrapolation.

In the second experiment, 3-month-olds were asked to extrapolate the rule "apparently nearer then bigger" with a sequential presentation. The habituation phase was exactly the same. The normal event was a bigger cube presented nearer, and the strange event was a smaller cube presented nearer. No difference in looking durations was observed between the normal and the strange event, but a significant difference appeared between the last habituation trials and the strange event, and not between the end of habituation trials and the normal event.

In the third experiment, a continuous movement in a 2D display was used to indicate to 4-month-olds the relation between the apparent distance and the size of an object. In the top left-hand corner of the background with perspective cues, a cube moved along a slanted line. When the cube apparently moved away, its size decreased, and when it apparently drew nearer, its size increased. When the infants were habituated to this situation, two events, a normal and a strange one, were presented alternately in the bottom right-hand corner. In the normal event, the movement was symmetrical to that of the habituation event, the cube was bigger when apparently nearer and smaller when apparently further. In the strange event, the movement was the same as in the habituation phase, but simply transferred forward to the bottom right-hand corner. As a consequence, the cube was bigger when apparently further and smaller when apparently nearer. No difference in looking durations appeared between the normal and the strange event, but a significant difference appeared between the last habituation trials and the strange event, not between the end of habituation trials and the normal event. 
These results do not indicate a clear mastery of the perspective rules but 3-month-olds and 4-month-olds seem to have used perspective cues in the 2D display. However, it can be noted that in these experiments as in the classic research, gaze direction is globally estimated by a human observer. Although the reliability of the indices produced is usually good, this procedure is not precise enough to determine exactly where infants are looking, and not sensitive enough to take into account short glances. Eye-tracking system allows to know if pertinent information is taken into account and if differences between the normal and the strange event are focused on the informative zones.

In using an eye-tracking system, one can know how infants take into account the visual information, if their exploration is based on the informative parts (the stimulus) or on the global information. Therefore, we decided to perform a replication of the third experiment in dynamic of Durand et al. (2003), using an eye tracking-system, the looking time data being completed by accurate gaze direction data. This system permits to check the gaze direction on the screen and more specifically at the figure during the animation.

In order to understand how the perspective representation emerges, we have tested again 4-month-old infants and older infants (5-month-olds) with the same animation of 2D display as in the third experiment of Durand et al. (2003) but using the eye-tracking system.

\section{Experiment 1}

\subsection{Participants}

The experiment was conducted on a sample of 29 full-term 4-month-old infants (17 male and 12 female, mean age -122 days, S.D. $=5$ days). The infants were recruited from the birth registry office in Paris and near Paris. The parents were contacted by mail and phone and they came to the laboratory where the infants were tested. 21 additional infants were observed but could not be used as participants because of crying (6), or failure to obtain good calibration (15).

\subsection{Apparatus}

The experimental system used was based on an ASL 504 Eye Tracking system with a specific software.

The system was monitored by a computer. In addition to the system and this computer (computer 1), a second computer (computer 2) was used to create the display presented to the subjects. Computer 2 was connected to a $50 \mathrm{~cm}$ monitor placed in front of the participants. The pan-tilt camera of the ASL system was placed at the top of this monitor, coupled with a scene camera that helped the experimenter to search for the infant's pupil at the beginning of the experiment. The participants were placed in an infant seat in front of the screen of computer 2 , in a $1 \mathrm{~m} \times 1 \mathrm{~m}$ box closed by a curtain. Computer 2 was connected to computer 1 and to ASL system. The first connection allowed computer 2 to be monitored by computer 1, to order the calibration points displayed and to begin the experiment at the end of the calibration phase. The second connection allowed computer 2 to record the $X, Y$ looking position, and the diameter of the pupil. The recording frequency of the eye position was of $50 \mathrm{~Hz}$. One gaze point was recorded every $20 \mathrm{~ms}$.

A dynamic reading program was used to read the files while displaying the experiment again. It indicated trial by trial all the gaze points detected by the system, the gaze points detected on the computer screen, the gaze points detected at the cube and $4^{\circ}$ around. This zone was traced at $4^{\circ}$ around the cube to take into account system error and participant saccade calibration error. With this program, the infant's gaze direction can be observed for as long as it is required.

The procedure comprised a calibration phase and an experimental one. The calibration phase allows to record the infant's gaze direction on a stimulus (a clown) in the upper-left corner and in the lower-right corner of the screen. These two calibration points are necessary to get some gaze direction data on the screen and the stimulus in the experimental phase.

\subsection{Animation}

On a computer monitor $(53 \mathrm{~cm})$, infants were habituated to a cube moving and taking different sizes in the upper left side of a room offering many perspective cues. In the point of view of an adult observer, the cube is moving from 
"front"1 to "back" and from "back" to "front" in the background with perspective lines. When the cube apparently moved away, its size decreased and when it apparently drew nearer its size increased (see Fig. 1). After habituation, the movement was presented on the lower right side. A normal and a strange event were presented alternately. In the normal event, the movement was symmetrical to that of the habituation event.

When an adult observed this situation, perspective laws were respected as in the habituation event (the cube was bigger when apparently nearer and smaller when apparently further) but with a change in the position of the cube path. In the strange event, the movement was the same as in the habituation phase, but simply transferred forward to the bottom right-hand corner. As a consequence, from an adult point of view, the cube was bigger when apparently further and smaller when apparently nearer and perspective laws were not respected even if the movement displayed was the same as in the habituation phase, with a translation.

A trial began when infants looked to the screen longer than $1 \mathrm{~s}$ and ended when they looked away for more than $1 \mathrm{~s}$. At the end of each trial, a blue curtain was drawn to signal the end of the animated event and the beginning of the next one. The subjects were considered to be habituated when the mean fixation time of the three last trials was less than $50 \%$ of the mean time in the first three trials. A maximum of 15 trials was used to avoid infants getting tired. The test events were presented alternately during six trials (three normal and three strange events).

Our hypothesis was that if 4-month-olds understand the perspective rule and are able to use perspective cues, they will look more at the strange situation than at the normal situation. If they do not, they will look more at the normal one, which is the newer one.

\subsection{Results}

First, analyses are carried out with 29 subjects' looks on the complete display.

As shown in Fig. 2, more looks were observed at the normal event than at the strange event but the difference was not significant $[F(1,27)=2.35 ; p=.137]$. They looked equally at the strange event and the last habituation trials. In the same way, they looked more at the normal event than at the last habituation trials but this difference was not significant $[F(1,27)=1.96 ; p=.172]$. A significant interaction of the trial factor with the following events: strange and last habituation trials, was observed. Infants' looks lowered as trials went along in the habituation phase and in the first two trials of the strange situation but increased in the last strange trial $[F(2,54)=4.91 ; p=.011]$.

Analyses were also carried out with 29 subjects' looks at the cube and $4^{\circ}$ around. Once again, as shown in Fig. 3 , the results indicate no significant difference between the gaze points in the strange event and in the normal event $[F(1$, $27)=2.49 ; p=.126]$. Nevertheless, a significant novelty reaction in the strange event was observed $[F(1,27)=5.74$; $p=.024]$ and in the normal event $[F(1,27)=5.84 ; p=.023]$. There were no other significant main effects or interactions.

\subsection{Discussion}

In this experiment, the difference between number looks on the whole screen in the strange and in the normal events was not significant as in the third experiment of Durand, Lécuyer, and Frichtel (2003) with 4-month-olds' looking time data recorded during this animation. No significant novelty reaction in the strange event or in the normal event was found on the complete display.

Infants' exploration of the cube indicates no significant difference between the looks for the normal and the strange events. Considering the looks at the cube, a novelty reaction in the strange event was found, as shown in Durand et al. (2003), but in this experiment the novelty reaction was also observed in the normal event.

A possible explanation of these results is that infants are too young to understand perspective cues in a $2 \mathrm{D}$ representation of 3D situation, as proposed by (Yonas \& Grandrud, 1985). One can think that perspective cues alone are not sufficient for 4-month-olds to perceive images as representations of 3D scenes. They might not have distinguished the strange event from the normal event. The lines forming the perspective were the only cue of depth but other cues must be necessary for 4-month-olds' representation. However, older infants may use these simple cues and therefore, the purpose of the second experiment was to test older infants with the same animation.

\footnotetext{
1 The word "front" and "back" are here under brackets because the distance of the stimulus is in fact the distance of the screen and remains always the same.
} 
Habituation phase (a)

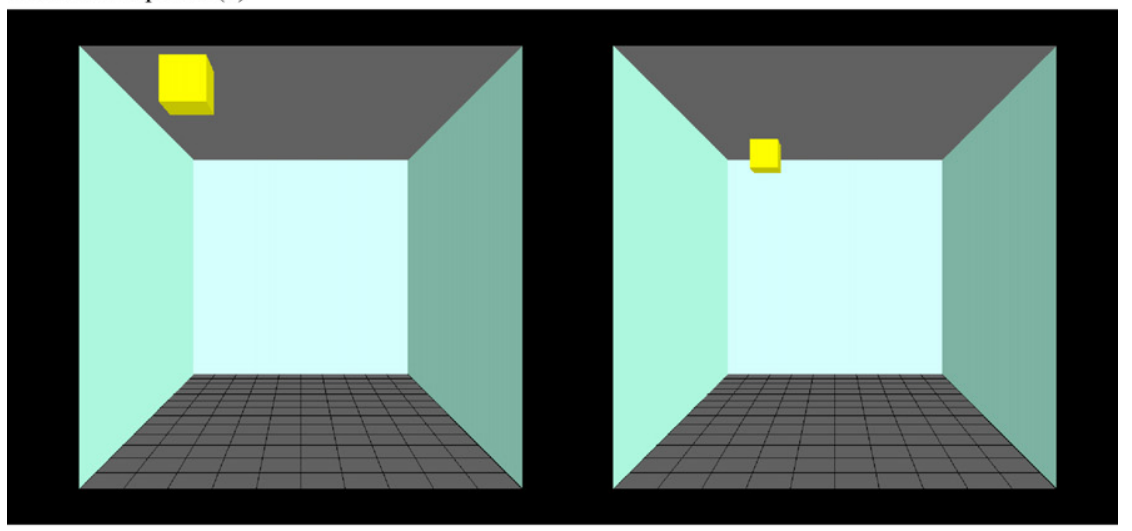

Normal Situation (b)

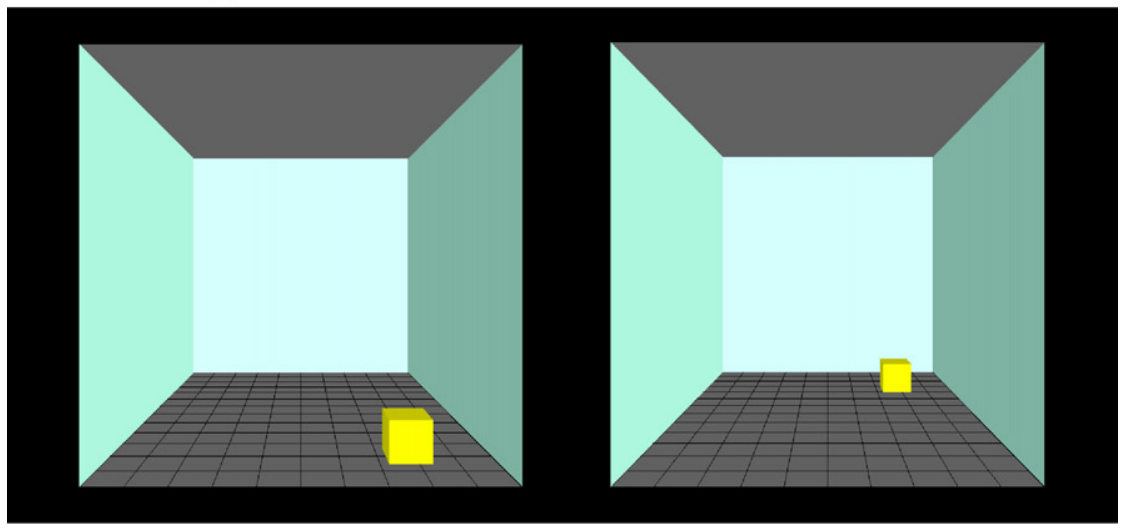

Strange Situation (c)

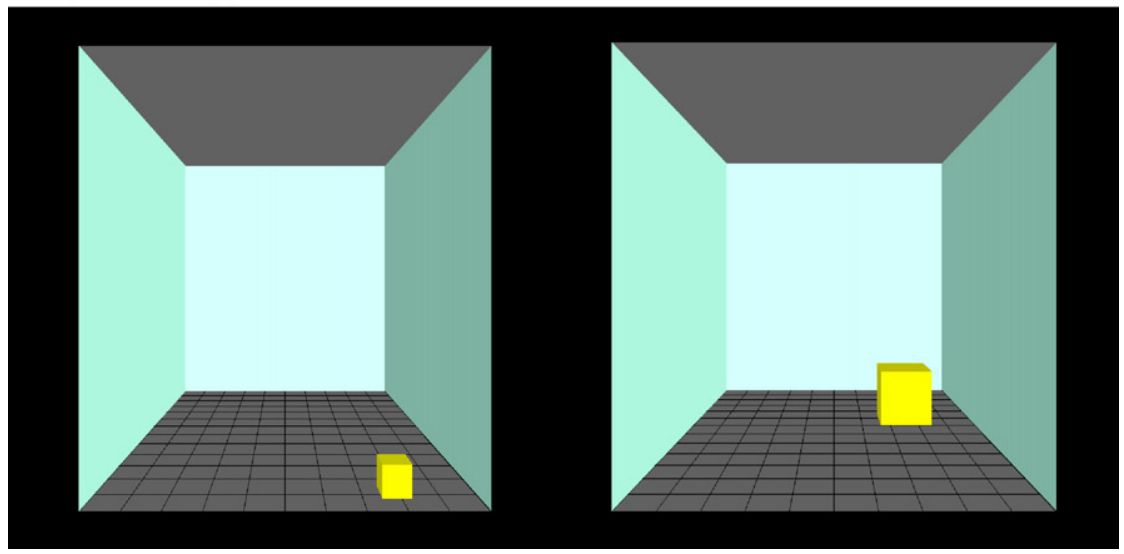

Fig. 1. Habituation phase, normal and strange situations. (a) Habituation phase, (b) normal situation, (c) Strange situation.

\section{Experiment 2}

\subsection{Participants}

The experiment was conducted on a sample of 25 full-term 5-month-old infants (14 male and 11 female, mean age -152 days, S.D. $=6$ days). The infants were recruited in the same way as in the first experiment. 21 additional 


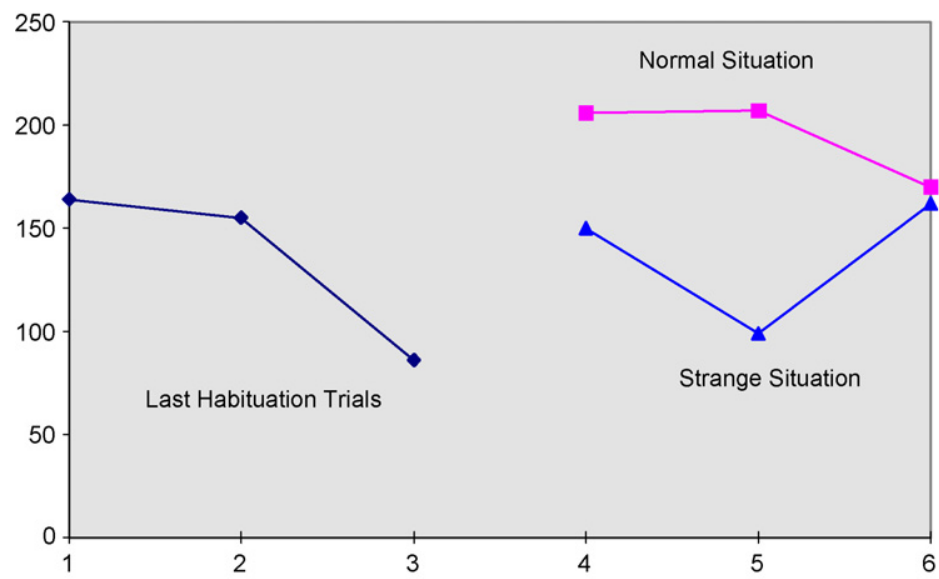

Fig. 2. Twenty-nine subjects' comparison looks on the screen during habituation phase and test phase.

infants were observed but could not be used as participants because of crying (6), or failure to obtain good calibration (15).

\subsection{Apparatus and procedure}

The apparatus, procedure and animation used in the second experiment were the same as those used in the first experiment.

\subsection{Results}

Analyses are carried out with 25 subjects' looks on the complete display.

As shown in Fig. 4, infants looked more at the normal event than at the strange event but the difference was not significant $[F(1,23)=.04 ; p=.836]$. A novelty reaction was observed in the strange event $[F(1,23)=6.29 ; p=.020]$ as in the normal event $[F(1,23)=4.43 ; p=.046]$.

An effect of the order of presentation was observed. Infants looked more at the test event when it began with the strange situation than when it began with the normal situation $[F(1,23)=5.91 ; p=.023]$.

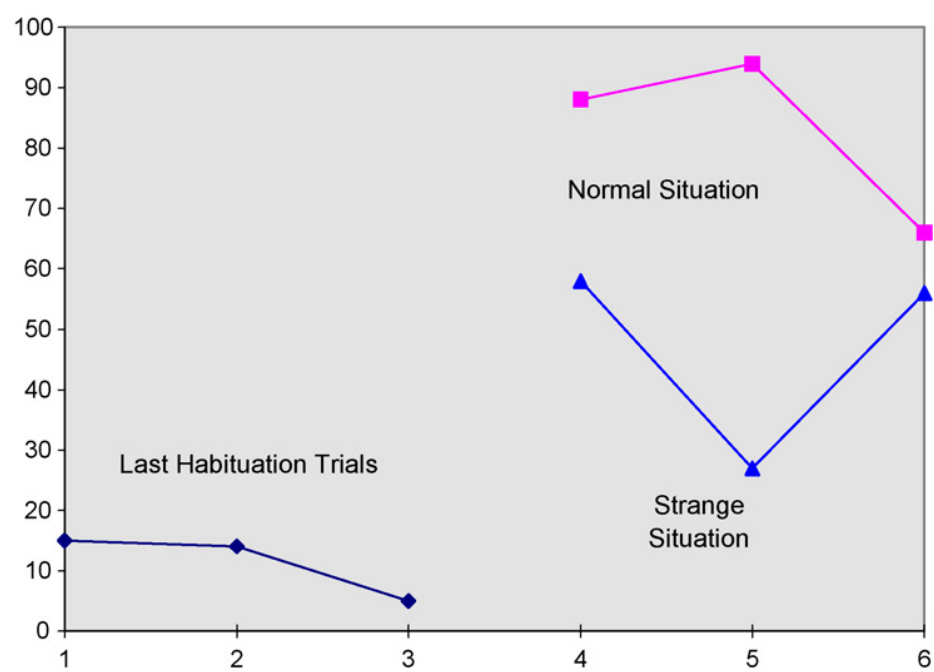

Fig. 3. Twenty-nine subjects' comparison looks at the cube and $4^{\circ}$ around during habituation phase and test phase. 


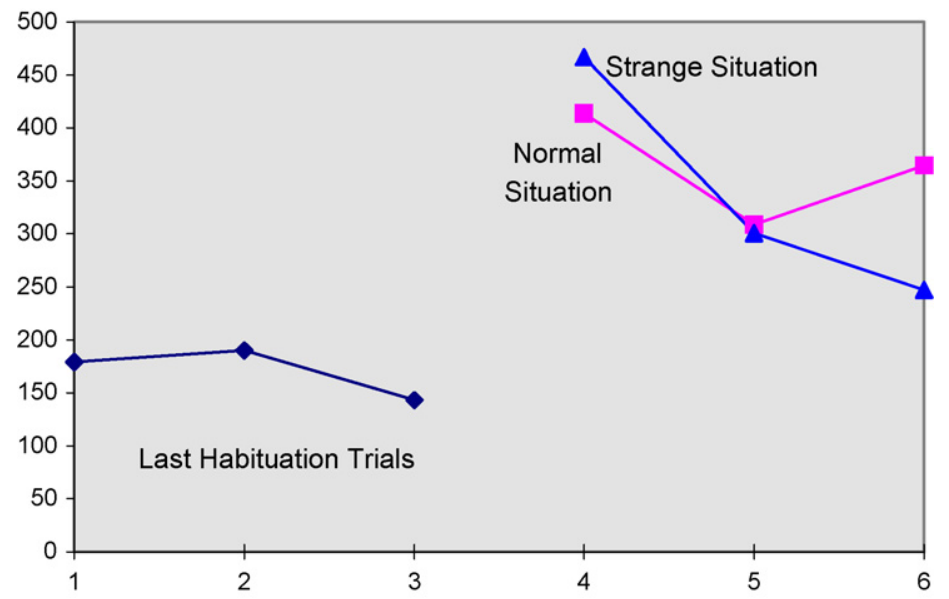

Fig. 4. Twenty-five subjects' comparison looks on the screen during habituation phase and test phase.

Other analyses were carried out with 25 subjects' looks at the cube and $4^{\circ}$ around and have shown the same results. No significant difference appeared in the looks for the normal and the strange event, even if they looked more at the strange event than at the normal event $[F(1,23)=.68 ; p=.417]$.

\subsection{Discussion}

In the second experiment, no significant difference was observed between looks data for the normal and the strange events on the whole screen, as with 4-month-olds in the first experiment. Analyses which were carried out with looks at the cube have shown the same results. Two hypotheses can be considered to explain the absence of difference between looks. The first one is that even if infants are five-month-olds, they are not always able to represent the perspective. They may be too young to have this ability and perhaps the experiment is not adapted to the infants' representation, which would be a reason for their preference for new events, as shown for 4-month-olds. A second hypothesis is that infants can be sensitive to the presentation order for the normal and the strange event.

In this second experiment, infants looked more at the two situation tests when the strange event was presented first. Such order effects are observed in several experiments in which the procedure of Kellman and Spelke (1983) was used (Durand \& Lécuyer, 2002; Poirier, Lecuyer, \& Cybula, 2000; Rovira \& Lécuyer, 1995).

The solution would be to change the procedure of Kellman and Spelke (1983) for a repeated presentation which is adapted of the "Partial-Lag" (Bertenthal, Haith, \& Campos, 1983). The partial-lag is a method for controlling spontaneous regression in the infant-control habituation paradigm. After habituation criterion has been reached by infants, two conditions are presented. One half of the infants are randomly assigned to a lag condition where they receive two postcriterion trials with the familiar stimulus before receiving the novel stimulus on postcriterion trials 3 and 4. For the other half infants, in the non-lag condition, the novel stimulus is presented immediately following criterion. Such a method allows the authors to control if infants' looking durations have increased in the postcriterion trials with spontaneous regression or not.

As in our experiments, two new events are presented in the test phase, strictly speaking, the partial-lag design cannot be used. Therefore, in our third experiment, we decided to use a modification of this paradigm: half of the infants were presented two strange events, followed by two normal events, and the other half received the opposite order of presentation: two normal events followed by two strange events.

\section{Experiment 3}

\subsection{Participants}

The experiment was conducted on a sample of 28 full-term 5-month-old infants (16 male and 12 female, mean age -152 days, S.D. $=5$ days). The infants were recruited in the same way as in the first and the second experiments. 


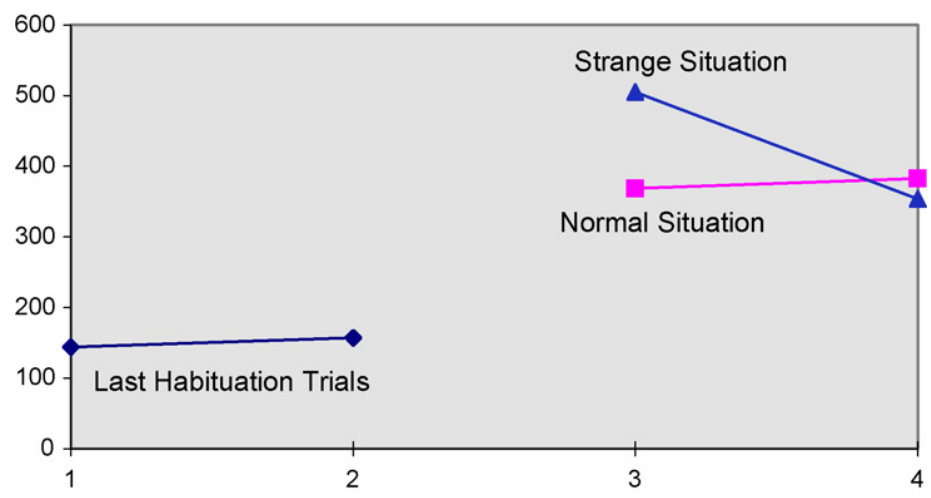

Fig. 5. Twenty-eight subjects' comparison looks on the screen during habituation phase and test phase.

23 additional infants were observed but could not be used as participants because of crying (7), failure to obtain good calibration (14) or software failure (2).

\subsection{Apparatus and procedure}

The apparatus used in the third experiment was exactly the same as in the previous experiments. The procedure comprised a calibration phase and an experimental phase.

After habituation, either the normal or the strange event was presented for two successive trials. Infants receiving the normal event first then saw the strange event for two trials. Infants receiving the strange event first were then shown the normal event for two trials.

\subsection{Results}

Analyses were carried out with 28 subjects' looks on the complete display.

As shown in Fig. 5, infants looked more at the strange event than at the normal event but the difference was not significant $[F(1,26)=1.27 ; p=.270]$. A novelty reaction was observed in the strange event $[F(1,26)=27.18 ; p=.00002]$ and also in the normal event $[F(1,26)=28.71 ; p=.00001]$.

The results show no effect of the order of presentation $[F(1,26)=1.81 ; p=.190]$.

Other analyses are carried out with 28 subjects' looks at the cube and $4^{\circ}$ around. As shown in Fig. 6, the results indicate that infants looked more at the strange event than at the normal event and this difference was significant $[F(1$, $26)=5.42 ; p=.028]$. An effect of the trials on the test was observed, looks lowered as trials went along $[F(1,26)=4.43$; $p=.045]$. The other results of this analysis were the same as those obtained in the analysis on the whole screen.

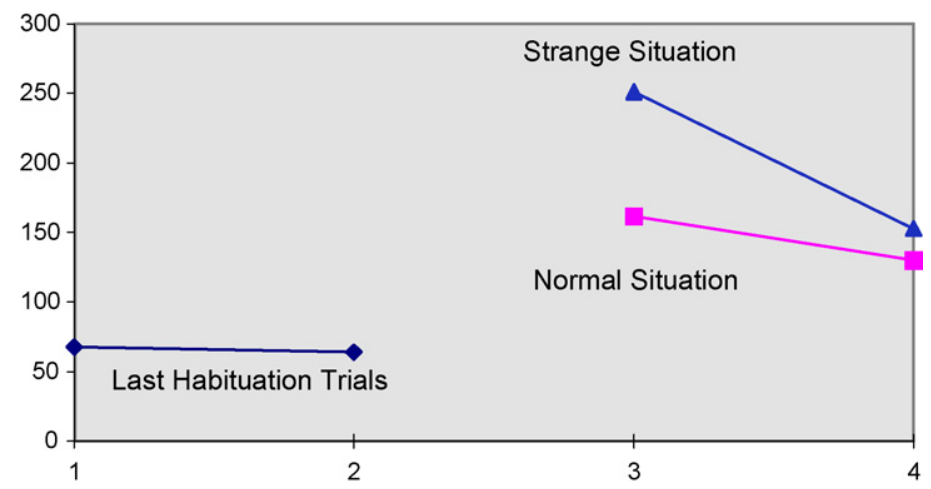

Fig. 6. Twenty-eight subjects' comparison looks at the figure and $4^{\circ}$ around during habituation phase and test phase. 


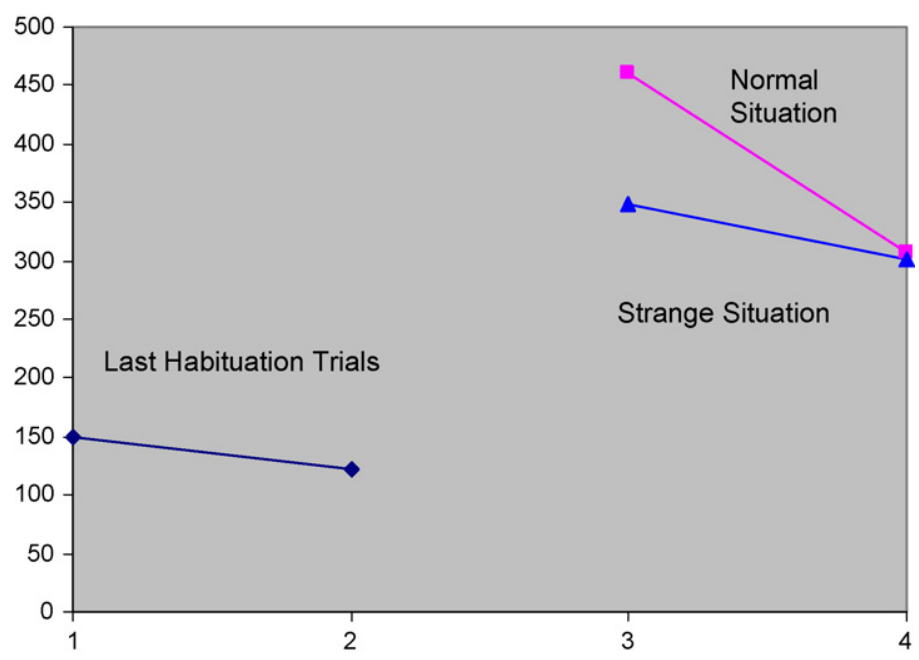

Fig. 7. Thirty-three subjects' comparison looks on the screen during habituation phase and test phase.

\subsection{Discussion}

In the third experiment, no significant difference between the looks for the normal and the strange event was observed on the complete displays. A novelty reaction was evidenced in the strange event but also in the normal event with looks on the screen. Infants' exploration on the cube indicates that 5-month-olds looked more at the strange event than at the normal event and this difference was significant. A novelty reaction in the strange event as in the normal event with looks at the cube was found again.

In eye tracking taking into account the most precise data concerning the cube, our hypothesis is supported: 5-montholds looked more at the strange event in which perspective laws were not respected, despite the fact that movement displayed followed a similar pathway to the habituation phase. They behaved as if they used perspective cues and understood the perspective rules. As in the two first experiments, infants also looked more at the strange and normal events than at the last habituation trials. Once more the test situation appeared to be new.

In order to draw any conclusions concerning 4-month-olds' ability to use the perspective cues, we decided to test them with the partial-lag variation in a fourth experiment.

\section{Experiment 4}

\subsection{Participants}

The experiment was conducted on a sample of 33 full-term 4-month-old infants (16 male and 17 female, mean age -121 days, S.D. $=4$ days). The infants were recruited in the same way as in the other experiments. 17 additional infants were observed but could not be used as participants because of crying (5), failure to obtain good calibration (10) or software failure (2).

\subsection{Apparatus and procedure}

The apparatus, procedure and animation used in the fourth experiment were the same as those used in the third experiment.

\subsection{Results}

Analyses were carried out with 33 subjects' looks on the complete display. 
As shown in Fig. 7, infants looked more at the normal event than at the strange event but the difference was not significant $[F(1,31)=.40 ; p=.533]$. A novelty reaction was observed in the strange event $[F(1,31)=6.14 ; p=.019]$ and also in the normal event $[F(1,31)=13.96 ; p=.001]$.

An effect of the trials between the normal situation and the last habituation trials was significant. Infants' looks lowered as trials went along $[F(1,31)=5.39 ; p=.027]$.

The results show no effect of the order of presentation $[F(1,31)=1.40 ; p=.246]$.

Other analyses are carried out with 33 subjects' looks at the cube and $4^{\circ}$ around and have shown the same results. No significant difference appeared in the looks for the normal and the strange event, even if they looked more at the normal event than at the strange event $[F(1,31)=0.21 ; p=.652]$.

One can note a significant interaction of the trials with the normal situation and the last habituation trials. Infants' looks lowered as trials went along $[F(1,31)=4.71 ; p=.038]$.

The other results of this analysis were the same as those obtained in the analysis on the whole screen.

\subsection{Discussion}

In this fourth experiment using a variation of the partial-lag, no significant difference was observed between number looks in the strange and in the normal events on the whole screen and at the cube as in the third experiment of Durand, Lécuyer, and Frichtel (2003) and as in our first experiment with 4-month-olds using a classical procedure.

On the contrary of the 5-month-olds who looked more at the strange situation than at the normal situation in our third experiment, no significant difference was observed in 4-month-olds.

A novelty reaction in the strange event was found with looks on the screen and at the cube, as shown in Durand et al. (2003) with looking time data, but in this experiment the novelty reaction was also observed in the normal event on the screen and at the cube. In our first experiment, with a classical paradigm, we found again in 4-month-olds these novelty reactions but only with looks at the cube (not on the complete display).

Thus, even in using a variation of the partial-lag paradigm in 4-month-olds, which appeared more adapted for 5month-olds, an ability of 4-month-olds to process the perspective cues with a 2D-display and to perceive depth cannot be evidenced.

The difference in the results between the 5-month-olds and 4-month-olds shows a development of their abilities with the age. Perhaps, perspective cues alone are not sufficient for 4-month-olds to perceive images as representations of 3D scenes.

\subsection{General discussion}

Four experiments were conducted in this study to understand how the depth representation emerges in infants. We have tested infants' ability to process the cues offered by perspective, in a dynamic 2D scene where "distance" was related to size.

In the habituation phase, a cube with a continuous movement was presented to indicate the relation between apparent distance and size of an object, in the upper left side of the screen. In the test phase, a normal and a strange event were displayed. In the normal event, the same rule was presented with a movement symmetrical to the habituation event in the bottom right side. For an adult, perspective laws were respected as in the habituation event but with a change in the cube trajectory (normal event). In the strange event, the movement was the same as in the habituation phase, but simply transferred forward to the bottom right-hand corner. As a consequence, for an adult, the cube was bigger when apparently further and smaller when apparently nearer and perspective laws were not respected although the movement displayed was the same as in the habituation phase, with a translation.

In the first experiment, 4-month-olds were tested with the eye-tracking system; this system takes into account the eye movements and the looking positions on the screen and on the stimulus during the animation. No difference in looking positions was observed, either when the looks on the all screen or at the cube were taken into account. These results were the same as in the research with looking durations of Durand et al. (2003).

This first experiment confirms overall results obtained by Durand et al. (2003). The more precise results about gaze directions obtained in this first experiment do not permit to conclude that 4-month-olds have used the perspective cues from our animation in the 2D display. The relation of the perspective laws does not seem to have been detected. Infants' exploration on the cube indicates a novelty reaction to the strange event as observed by Durand et al. (2003), but a 
novelty reaction was also observed with the normal event. Maybe in that situation infants consider the two events as new ones, in fact they are surprised that the location of the movement was changed and therefore do not take into account the difference between the new but normal event and the new but strange event. This may be due to the fact that depth cues in our animation (lines' perspective) have been insufficient for 4-month-olds to represent the depth from a 2D display.

In the second experiment, 5-month-olds were tested with the same paradigm and animation. No difference in looking positions appeared between the normal and the strange event, either on the whole screen or only at the cube. A significant difference appeared between number of looks at the whole screen for the last habituation trials and the strange event and for the last habituation trials and the normal event. The same difference was considered in the looks at the cube. 5-month-olds reacted to the novelty test events either normal or strange. However, an order effect was observed: when the test began with the strange situation, both events received more looks than the last habituation trials. When the test began with the normal event, no novelty reaction was observed.

To avoid this order effect, in the third experiment, 5-month-olds were tested with a repeated presentation, which is an adaptation of the "Partial-Lag Design" (Bertenthal et al., 1983), and the same animation. When taking into account looks at the cube, infants looked more at the strange event than the normal event (this difference existed with looks on the whole screen but it was not significant). No order effect was observed. A significant difference appeared between the last habituation trials and the strange event, as between the last habituation trials and the normal event. Once again, infants reacted to both novelty test events, but in addition, 5-month-olds seem to have been surprised by the strange event, which did not respect the perspective laws.

To draw any conclusions with 4-month-olds, in the same experimental conditions that 5-month-olds, they were also tested with the variation of the Partial-Lag paradigm and the same animation in a fourth experiment. No difference in looking positions appeared between the normal and the strange event, either on the whole screen or only at the cube, but a significant difference appeared between number of looks at the whole screen and at the cube for the last habituation trials and the strange event and for the last habituation trials and the normal one. 4-month-olds reacted to the novelty test events either normal or strange. No order effect was observed. On the contrary of the 5-month-olds, perspective cues alone are not perhaps sufficient for 4-month-olds to perceive images as representations of 3D scenes.

Despite the difference in age, results concerning looks on the whole screen and at the cube were nearly similar in the first two experiments. Despite the similarity of age of the infants in experiments 2 and 3, the difference in paradigm produced some differences in number of looks at the strange event. This difference was significant only for the number of looks at the cube. Using an eye-tracking system allows evidencing differences in looks that the usual procedures do not permit to evidence.

Thus, this research demonstrated the ability to process perspective cues with a 2D display in 5-month-olds when perspective is the only depth cue provided. However, this capacity is not proved in 4-month-olds, either a classical paradigm in experiment 1 or a variation partial-lag paradigm in experiment 4 . Results obtained with 4-month-olds are compatible with those obtained by Durand et al. (2003).

Durand and Lécuyer (2002) demonstrated with a 2D replication of the research of (Baillargeon et al., 1985) that 4-month-olds understand object permanence and dynamic interposition when perspective cues are present, while Lécuyer and Durand (1998) did not find the same results when infants are not presented with any perspective cues in an object permanence situation with a 2D replication of the research of Baillargeon and Graber (1987). These experiments showed the importance of perspective cues for infants when they are displayed with interposition cues, but the experiments presented in the present research show the importance of perspective cues for infants when presented alone, in a situation where the size of an object depends on its apparent distance.

Five-month-olds can use perspective cues alone in a 2D display and these cues seem sufficient to represent the depth, whereas 4-month-olds did not master them well enough to give clear-cut indices of this use from our animation. The results of Slater et al. (1990) demonstrated that, at birth, infants can take into account the relation between size constancy and distance in a 3D situation but it is not so evident in a 2D display.

The sensitivity to the static pictorial information was shown in a lot of researches in the literature. Infants' responsiveness to static pictorial information was evidenced in 7-month-olds and in 6-month-olds by Yonas et al. (1978) and Yonas and Owsley (1987) but was not found in 5-month-old-infants' groups. However, a longitudinal study showed a variability in the age at which infants started using perspective cues and texture gradients for perceiving depth (22-28 weeks of age) and their sensitivity emerged across 2-8 weeks (Yonas et al., 2002). 
Nevertheless, kinetic pictorial information can help younger infants to represent depth from a dynamic scene, as in our experiment with the continuous movement of the cube.

Movement makes perception easier. Indeed, several studies have shown that 4-month-old infants are able to perceive object unity if the visible parts of the centre-occluded rod share a common motion but not if they are confronted with a stationary rod-and-box display (see Kellman \& Spelke, 1983; Kellman, Spelke, \& Short, 1986). Kellman and Spelke employed three-dimensional displays. Later, these findings were extended to a two-dimensional object unity configuration in 4-month-olds under some display conditions as in using common motion of two visible aligned rods in front of a textured background (Johnson \& Aslin, 1996, 2000), and in 2-month-olds if more of the rod is visible behind the occluding box than in traditional displays (Johnson \& Aslin, 1995).

The procedure used in our experiments has to be discussed. In the second experiment, 5-month-olds are sensitive to the presentation order of the normal and the strange event. They looked more at the test events (normal or strange event) when the strange situation was presented first. Comparable order effects were observed in several experiments when the procedure of Kellman and Spelke (1983) was used (Durand \& Lécuyer, 2002; Poirier et al., 2000; Rovira \& Lécuyer, 1995). After the habituation phase, if the event presented is novel and strange, the interest of the infants is more aroused than if it is only novel, and therefore, they more actively compare the normal to the strange events. Looks are therefore longer for both.

In using a procedure with a presentation repeated which is adapted of the "Partial-Lag Design" (Bertenthal et al., 1983) in the third and fourth experiments, no effect of the order of presentation was found. The test events were presented for four trials with a sequence of two normal events and two strange events, and not alternately.

This procedure permits to find again the tendencies of the results obtained in 4-month-olds with a classical paradigm and reinforce these appeared in 5-month-olds. Four-month-olds tend again to looked more at the normal situation while 5-month-olds looked more at the strange situation. The difference between the results of the 4-month-olds and 5 -month-olds with this procedure and the same animation is more salient.

The capacity to understand a 2D display representing a 3D scene and to extract depth cues corresponds to a depth representation. 5-month-olds seem to be able to use the perspective cues alone to detect that the size of an object varies as a function of its apparent distance; they seem to have mastered the use of cues and to have perceived a depth representation. In 4-month-olds, perspective cues in this animation alone did not seem to be sufficient for perceiving depth. The use of this type of cue is still delicate in 4-month-olds. In another research, Johnson and Aslin (1996) found that 4-month-old infants who viewed a partial occlusion displays of a rod and box with no background texture, or displays in which the rod pieces were non-aligned but relatable looked equally at the two posthabituation displays (one rod or two rod piece). Infants who viewed displays containing nonrelatable rod pieces looked longer at the complete rod, implying that nonrelatable edges specify disjoint objects. They conclude that multiple visual cues are necessary for the perception of object unity (motion of display elements, depth ordering, edge orientations ...) but not individually efficient.

In literature, the role of perspective as a depth cue is less studied, but it would be interesting to better understand this perception in young infants, when perspective is presented alone or with other cues of depth in a dynamic 2D display.

\section{References}

Baillargeon, R., \& DeVos, J. (1991). Object permanence in young infants: Further evidence. Child Development, 62(6), $1227-1246$.

Baillargeon, R., \& Graber, M. (1987). Where's the rabbit? 5,5-month-old infants' representation of the height of a hidden object. Cognitive Development, 2(4), 375-392.

Baillargeon, R., Spelke, E. S., \& Wasserman, S. (1985). Object permanence in five-month-old infants. Cognition, 20, $191-208$.

Bertenthal, B. I., Haith, M. M., \& Campos, J. J. (1983). The partial-lag design: A method for controlling spontaneous regression in the infant-control habituation paradigm. Infant Behavior and Development, 6, 331-338.

Campos, J. J., Langer, A., \& Krowitz, A. (1970). Cardiac responses on the visual cliff in prelocomotor human infants. Science, $170,196-197$.

Durand, K., \& Lécuyer, R. (2002). Object permanence observed in four month-old-infants with a 2D display. Infant Behavior \& Development, 25(3), 269-278.

Durand, K., Lécuyer, R., \& Frichtel, M. (2003). Representation of the third dimension: The use of perspective cues by 3-and 4-month-old infants. Infant Behavior \& Development, 26, 151-166.

Fantz, R. L. (1961). The origin of form perception. Scientific American, 204, 66-72.

Fantz, R. L., \& Nevis, S. (1967). Pattern preference and perceptual-cognitive development in early infancy. Merrill Palmer Quaterly, 13, 77-108.

Gibson, E. J., \& Walk, R. D. (1960). The “visual cliff”. Scientific American, 202, 64-71. 
Granrud, C. E., Yonas, A., \& Pettersen, L. (1984). A comparison of responsiveness to monocular and binocular depth information in 5- and 7-month-old infants. Journal of Experimental Child Psychology, 38, 19-32.

Johnson, S. P., \& Aslin, R. (1995). Perception of object unity in 2-month-old infants. Developmental Psychology, 31, 739-745.

Johnson, S. P., \& Aslin, R. N. (1996). Perception of object unity in young infants: The roles of motion, depth, and orientation. Cognitive Development, 11, 161-180.

Johnson, S. P., \& Aslin, R. N. (2000). Infants' perception of transparency. Developmental Psychology, 36, 808-816.

Kellman, P. J., \& Spelke, E. S. (1983). Perception of partly occluded objects in infancy. Cognitive Psychology, 15(4), $483-524$.

Kellman, P. J., Spelke, E. S., \& Short, K. R. (1986). Infant perception of object unity from translatory motion in depth and vertical translation. Child Development, $57,72-86$.

Lécuyer, R., \& Durand, K. (1998). Bi-dimensional representations of the third dimension and their perception by infants. Perception, 27(4), 465-472.

Lécuyer, R., Pêcheux, M.-G., \& Streri, A. (1994). Le développement cognitif du nourrisson. I Paris: Nathan.

Mandler, J. M. (1998). Representation. In W. Damon, D. Kuhn, \& R. S. Siegler (Eds.), Handbook of child psychology. New York: Wiley.

Maya, G. S., Yonas, A., \& Knill, D. C. (2001). Development of infants' sensitivity to surface contour information for spatial layout. Perception, 30, 167-176.

Nanez, J. S., \& Yonas, A. (1994). Effects of luminance and texture motion on infant defensive reactions to optical collision. Infant Behavior \& Development, 17, 165-174.

Poirier, C., Lécuyer, R., \& Cybula, C. (2000). Categorization of geometric figures composed of three or four elements by 3-month-old infants. Cahiers de psychologie cognitive, 19(2), 221-244.

Rovira, K., \& Lécuyer, R. (1995). Organisation perceptive chez les bébés de 4 mois: la détection de permutations. Enfance, 2, $135-146$.

Slater, A. M., Mattock, A., \& Brown, E. (1990). Size constancy at birth: Newborn infants' responses to retinal and real size. Journal of Experimental Child Psychology, 49, 314-322.

Yonas, A., \& Arterberry, M. E. (1994). Infants perceive spatial structure specified by line junctions. Perception, 23, $1427-1435$.

Yonas, A., Arterberry, M. E., \& Granrud, C. E. (1987). Space perception in infancy. Child Development, 4, 1-34.

Yonas, A., Cleaves, W., \& Pettersen, L. (1978). Development of sensitivity to pictorial depth. Science, 200, 77-79.

Yonas, A., Elieff, C. A., \& Arterberry, M. E. (2002). Emergence of sensitivity to pictorial depth cues: Charting development in individual infants. Infant Behavior \& Development, 25, 495-514.

Yonas, A., \& Grandrud, C. E. (1985). Development of the depth sensitivity in infants. In J. Mehler \& N. Fox (Eds.), Neonate cognition: beyond the blooming, buzzing confusion. Hillsdale, NJ: Erlbaum.

Yonas, A., \& Granrud, C. E. (1985). Development of visual space perception in young infants. In J. Mehler \& R. Fox (Eds.), Neonate cognition: Beyond the blooming, buzzing confusion (pp. 45-67). Hillsdale, NJ: Erlbaum.

Yonas, A., \& Owsley, C. (1987). Development of visual space perception. In P. Salapatek \& L. Cohen (Eds.), Handbook of infant perception: From perception to cognition (pp. 80-122). New York: Academic Press. 